\title{
Chip emissivity mapping for blue and white light emitting diodes
}

\author{
by F. Belfio ${ }^{a, b}$, J.-F. Veneau ${ }^{b}$ and O. Fudym ${ }^{a}$ \\ a Univ. Toulouse; Mines Albi; CNRS; Centre RAPSODEE, Campus Jarlard, F-81013 Albi, France, \\ olivier.fudym@mines-albi.fr, fiona.belfio@mines-albi.fr \\ b LED Engineering Development, jean-francois.veneau@led-development.fr
}

\begin{abstract}
In order to measure the temperature distribution at the chip surface of blue and white light-emitting diodes, we used two temperature conventional emissivity measurement method, which consists in processing the thermographic signal for two uniform controlled temperatures $T_{1}$ and $T_{2}$. In this study, we report the evolution of emissivity with increasing temperature between $20^{\circ} \mathrm{C}$ and $80^{\circ} \mathrm{C}$ from to the ambient temperature, about $20^{\circ} \mathrm{C}$.
\end{abstract}

\section{Introduction}

High brightness light-emitting diode (LED) is a promising technology for lighting. The light performance of LED is affected by a series of thermal problems, which one major source is the semiconductor chip. Infrared-thermography is used to measure temperature distribution of the chip. However, precise temperature mapping over the chip is influenced by many factors, including emissivity. LED chips in charge can easily reach $80^{\circ} \mathrm{C}$. In this study, we calculated the emissivity for blue and white chips for different temperatures by a conventional method.

\section{Light emitting diodes}

\subsection{Generality}

This paper discusses high power light emitting diodes. This technology evolves quickly. Nowadays the luminous efficacy for a single device is about $120 \mathrm{Im} / \mathrm{W}$, while in1995 the luminous efficacy is $20 \mathrm{Im} / \mathrm{W}$. The luminous efficiency defines the ability of a light to produce a significant visible flow from one electrical watt consumed. The power efficiency is defined as Eq. (1):

$$
\eta_{\text {power }}=P_{\text {opt }} / P_{\text {gles }}
$$

where $P_{\text {opt }}$ is the optical power emitted into free space and $P_{\text {elec }}$ is the electrical power provided to the LED. Following $P_{\text {elec }}, \eta_{\text {power }}$ ranges between 20 and $35 \%$. The electrical power that is not transformed optically becomes heat. The light is emitted by the $p-n$ junction. The light extraction efficiency can be enhanced by using dome-shaped encapsulants with a large refractive index.

The study is about LED that generates white light; it means a LED that is composed a blue chip plus a yellow phosphor. The blue chip will be also studied. The dimensions are similar for white and blue chips (figure 1).
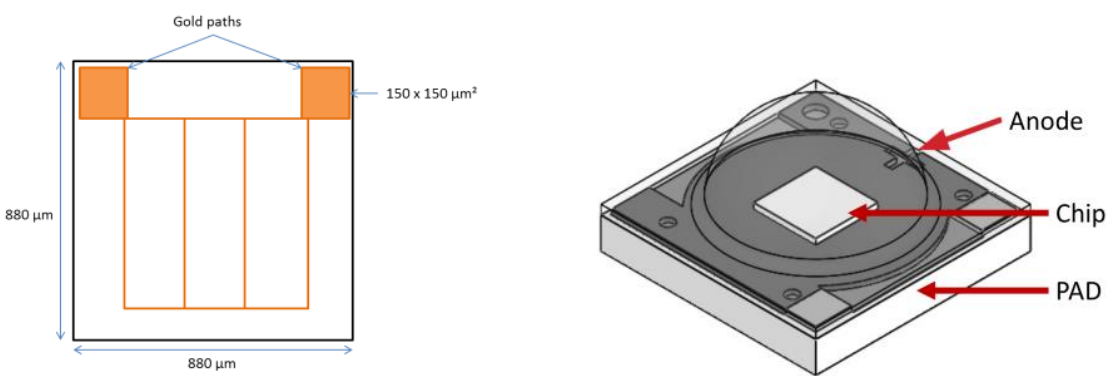

Fig. 1. Schema of a chip and a LED

\subsection{Junction temperature of light emitting diodes}

The junction temperature is a critical parameter [1]. The junction temperature is relevant for several reasons. Firstly, the internal quantum efficiency depends on the junction temperature. Secondly, high temperature operation shortens the device lifetime. Thirdly, a high device temperature can lead to degradation of the encapsulant. 
In the context of our research, we want to know the junction temperature at each point of the chip. To obtain this, we are going to study the temperature distribution at the chip surface of blue and white light-emitting diodes thanks the infrared thermography. Thermal measurements using infrared thermography was also used [2] to detected local hot spots due to high electric stress.

To have an accurate infrared measurement, we are looking to the chip emissivity on the temperature range of LED use $\left(20\right.$ and $\left.80^{\circ} \mathrm{C}\right)$. The calculated emissivity will be a directional emissivity for the spectral range of the infrared camera. To facilitate the measurements, the study will focus on diodes without dome, because the encapsulant is semitransparent in the infrared spectrum.

\section{Emissivity measurements}

\subsection{Emissivity measurement methods}

This conventional emissivity measurement method [3-4] consists of recording thermal images when the sample is heated uniformly to a known temperature $T_{2}$. The $T_{1}$ temperature is the ambient temperature. The area where it is desired to measure the emissivity is compared to a reference area assumed as a known emissivity region such as a black body signal. The calculation of the emissivity can be described with the following equation:

$$
\varepsilon_{i j}=\frac{J_{i j} 2-J_{i j} 1}{J_{b, T_{2}}-J_{b, T_{1}}}
$$

Where:

- $l_{i j} 1$ and $l_{i j} 2$ correspond to signals obtained by the infrared camera of the sample at temperature $T_{1}$ and $T_{2}$.

$-\varepsilon_{\mathrm{i} j}$ emissivity of area $i j$.

and $\mathrm{T}_{2}$ respectively.

- $J_{b, T_{1}}$ and $J_{b, T_{2}}$ correspond to signals obtained by the infrared camera of the black body at temperature $\mathrm{T}_{1}$

\subsection{Experimental}

In this case, the goal is to obtain the chip emissivity mapping for blue and white LED, switched off. Two samples were studied: blue and white light-emitting diodes soldered on a printed circuit board (PCB). A part of the PCB, near the LED was painted with a black paint. A Peltier device is used to heat the samples. Temperature is measured with different ways: with a type $T$ thermocouple and the infrared measurement of a black paint (Fig.2), with an emissivity between 0.95 and 0.98 . We assume that the emissivity of the black paint is equal to 1 .

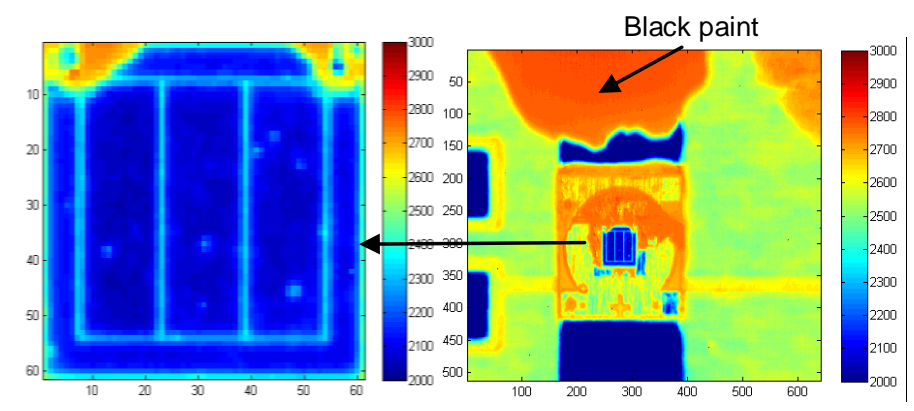

Fig. 2 Infrared image of blue chip

The ambient temperature $\mathrm{T}_{1}$ is about $20^{\circ} \mathrm{C}$. The infrared images were taken between $20^{\circ} \mathrm{C}$ and $80^{\circ} \mathrm{C}$, about every $3^{\circ} \mathrm{C}$. Emissivity will be calculated for each temperature. To measure the thermal radiation distribution on the LED surface [5], we used an InSb FPA infrared camera (640x512) and a G1 lens manufactured by FLIR Systems Inc. The infrared microscope system has a spectral response in the 3.5-5.1 $\mu \mathrm{m}$ range.

\section{Results : emissivity and temperature mapping}

For each studied LED (white and blue chips), the power efficiency is calculated for various values of the input electrical power. The luminous flux was measured thanks a spectrometer and an integrating sphere. The measured luminous flux is compared the electrical power injected. 


\subsection{Blue chips}

\subsubsection{Emissivity mapping of blue chip}

For each temperature, the mean emissivity has been calculated on the central part of chip (figure 3). Moreover emissivity has been calculated for each pixel. The figure 4 shows the cartography of emissivity for two different values of the uniform temperatures $\mathrm{T}_{2}=40^{\circ} \mathrm{C}$ and $\mathrm{T}_{2}=79^{\circ} \mathrm{C}$. The emissivity of the blue chip is about 0.49 . Normally the emissivity of the gold path would be lower than the chip. The dimensions of the gold paths are too small for the resolution of the camera: thickness $=10 \mu \mathrm{m}$ and height $=3 \mu \mathrm{m}$.

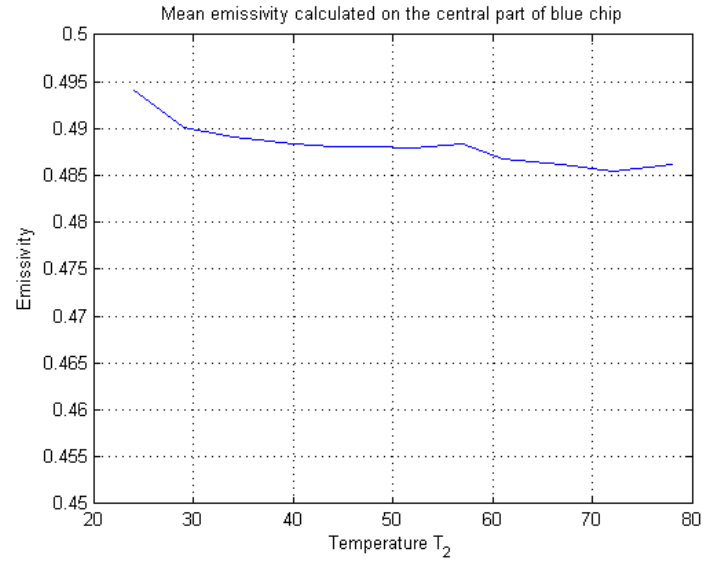

Fig. 3. Constant emissivity with temperature $T_{2}$
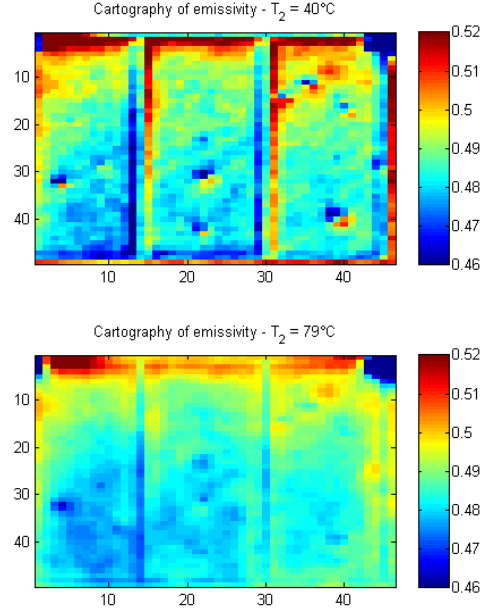

Fig. 4. Cartography of emissivity

\subsubsection{Temperature mapping of blue chip}

We apply this emissivity of infrared images of the same blue chip. Figure 5 shows the temperature when the LED is switched off. The mean temperature is equal to the ambient temperature. Figure 6 shows a LED that is supplied by an electrical power of $3,65 \mathrm{~W}$, the mean temperature increases until $68^{\circ} \mathrm{C}$. There is a more significant overheating near the gold paths. For the next infrared images, the size of a pixel is equal to $11 \mu \mathrm{m}$.

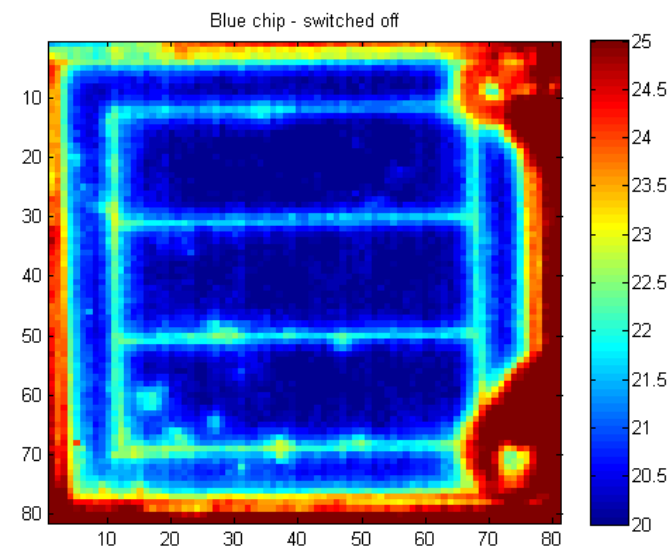

Fig. 5. Temperature distribution at the blue chip surface of switched off $L E D$

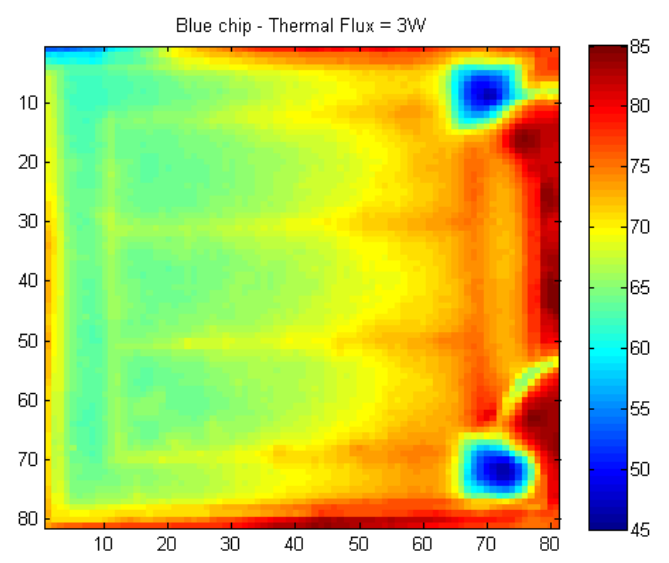

Fig. 6. Temperature distribution at the blue chip surface that is supplied at 3.65W and that generates $3 \mathrm{~W}$ of heat 


\subsection{White chips}

\subsubsection{Emissivity mapping of white chip}

The method is similar for the white chip. The gold paths are less apparent because of the phosphor and the emissivity is different (figure 7). The emissivity for the white LED's chip is found to be 0.82

Cartography of emissivity $-T_{2}=45^{\circ} \mathrm{C}$

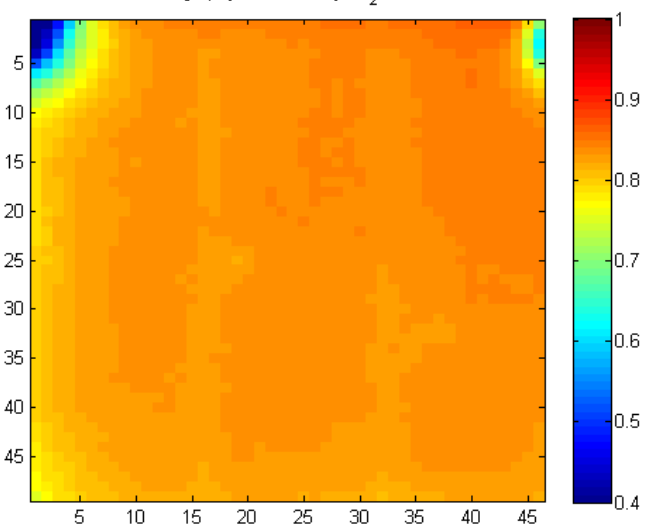

Fig.7. Cartography of emissivity for a white chip

\subsubsection{Temperature mapping of white chip}

After applying the emissivity map, we obtain the temperature field, as shown in figure 8 when the white LED is switched off and in figure 9 when this LED is turned on with 3,70 W input power. The temperature is uniform over the surface of the white chip. We obtain a mean temperature about $67,3^{\circ} \mathrm{C}$. We notice a significant overheating near the gold path at the bottom right of the image. There is a difference of temperature equal to $15^{\circ} \mathrm{C}$ between the bottom right corner and the top left corner of figure 9.

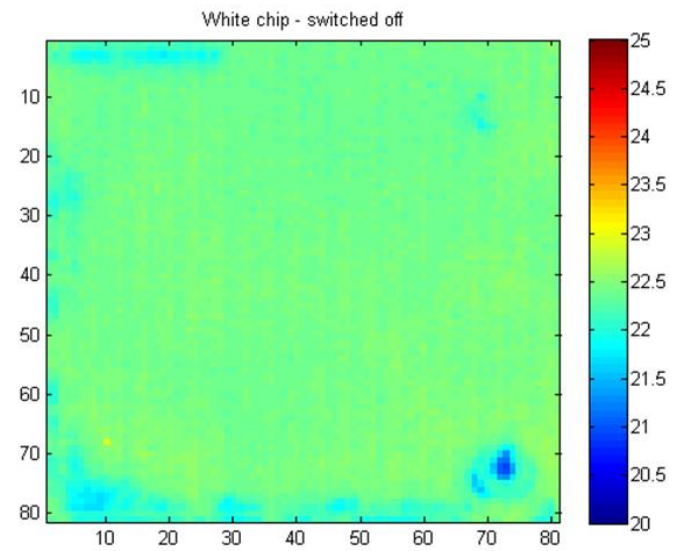

Fig. 8. Temperature distribution at the white chip surface of switched off LED

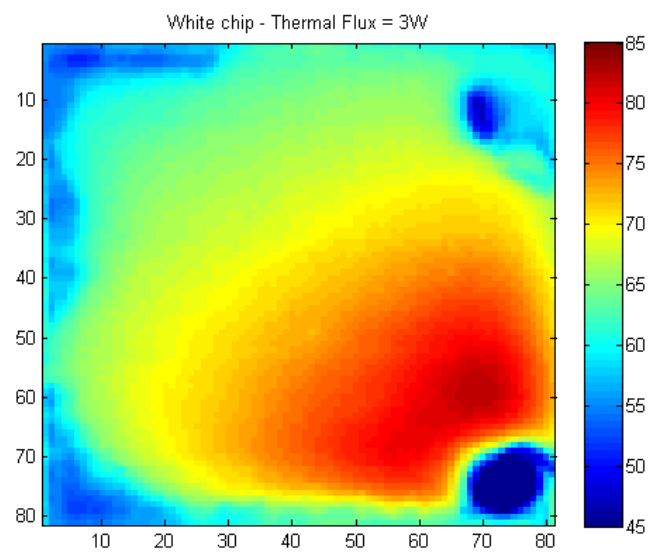

Fig. 9. Temperature distribution at the white chip surface that is supplied at 3.70W and that generates $3 \mathrm{~W}$ of heat

\subsection{Temperature versus the electrical power}

Infrared thermography measurements have been done for a white chip and a blue chip. We varied the supply current from 0 to $1.1 \mathrm{~A}$, with an increment of 0,1A. For each current, the average of 100 infrared images was computed in order to increase the signal-to-noise factor. The voltage has been also measured, what permits to calculate the electrical power provided to the LED. 
Figure 10 shows the increase of the mean temperature versus the electrical power. The blue chip and the white chip have the same behavior, and the temperature difference is quite low, as shown in Figure 10b, where there is an average offset of $1,7^{\circ} \mathrm{C}$ between the blue chip and the white chip.

It has been reported that the phosphor temperature becomes the highest in the LED packages rather than the junction temperature $[6,7]$. Apparently, in our case, the phosphor temperature and the junction temperature are near because we have not studied a normal packaging of LED. We removed the encapsulant dome which is normally above the chip (Fig. 1).

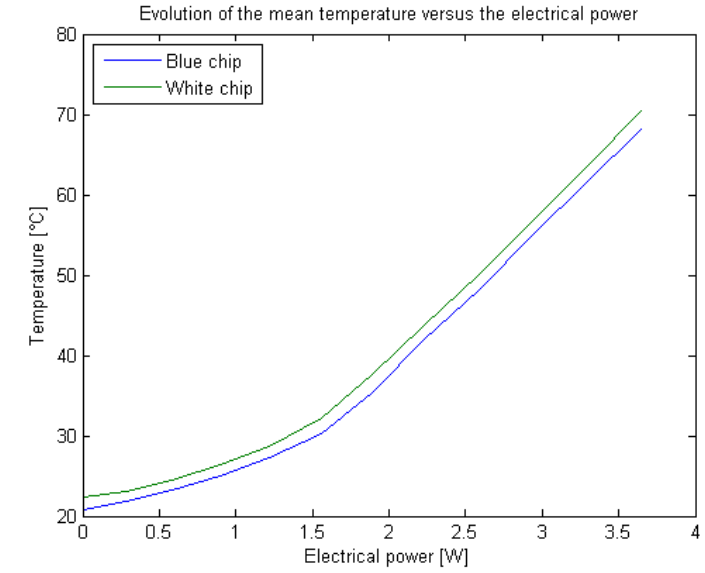

Fig. 10. a Increase of the mean temperature with the input electrical power

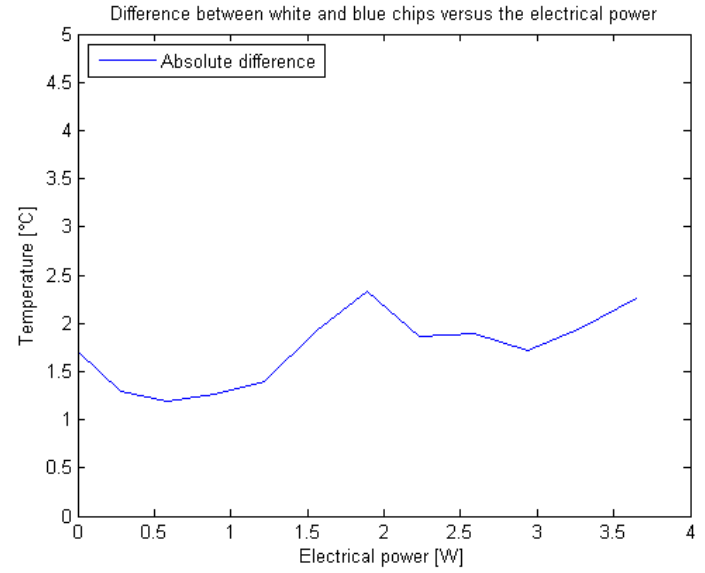

Fig. 10. $\boldsymbol{b}$ Temperature difference between the blue chip and the white chip

\section{Conclusion}

The emissivity for a blue chip is about 0,48 and for a white chip is 0,82 . These measurements will allow to have accurate results on the surface temperature of the chips. The temperature distributions become more heterogeneous when the input electrical power of the LED increases. Further, the temperature difference remains low between the chips with and without phosphor.

\section{Acknowledgement}

The authors would like to thanks the company LED Engineering Development and the laboratory RAPSODEE of Mines Albi for support and funding.

\section{REFERENCES}

[1] Schubert E.F., "Light-emitting diodes", Cambridge University Press, Second Edition, pp. 101-111, 2006.

[2] Wang, C., Chen, T., Fu, H., Chang, T., Chou, P., \& Chu, M., "Analysis of thermal characteristics and mechanism of degradation of flip-chip high power LEDs", Microelectronics Reliability, vol. 52, pp. 698-703, 2012

[3] Walach T., "Emissivity measurements on electronic microcircuits". Measurement, vol. 41, pp. 503-515, 2008.

[4] Boue C., Fournier D., "Cost-effective infrared thermography protocol for $40 \mathrm{~lm}$ spatial resolution quantitative microelectronic imaging ", Infrared Physics \& Technology, vol. 48, pp. 122-129, 2006.

[5] Chang K.S., Kim J.-Y., Kook M. H., Ryu S.Y., Yang S.C., Kim G. H. Quantitaive infrared micro-thermography of light emitting diodes", Proceedings of 11th Quantitative InfraRed Thermography conference, Naples (Italy), 2012.

[6] Yan, B., Tran, N. T., You, J. \& Shi, F. G., "Can Junction Temperature Alone Characterize Thermal Performance of White LED Emitters ?", vol. 23, 555-557, 2011

[7] Luo, X. \& Hu, R., "Calculation of the phosphor heat generation in phosphor-converted light-emitting diodes", International Journal of Heat and Mass Transfer, vol. 75, 213-217, 2014 